\title{
Le cycle de l'eau dans le système de mousson d'Afrique de l'Ouest
}

\author{
Christophe Peugeot(1), Olivier Bock(2), Aaron Boone (3), \\ Bernard Cappelaere( $(1)$, Marielle Gosset(4), Rémi Meynadier(5), \\ Luc Séguis(1), Thierry Lebel(6) et Jean-Luc Redelsperger(7) \\ (1) IRD, HSM, Université Montpellier 2 - CC MSE \\ Place Eugène Bataillon - 34095 Montpellier \\ (2) IGN, LAREG, Champs-sur-Marne, France; GRGS \\ (3) CNRS, CNRM/GAME, Toulouse, France \\ (4) IRD, GET, Toulouse, France \\ (5) CNRS, LATMOS, Paris, France \\ (6) IRD, LTHE, Grenoble, France \\ (7) CNRS, LPO, Technopôle Brest-Iroise, Plouzane, France
}

\section{Résumé}

L'amélioration de la compréhension du cycle de l'eau dans le système de la mousson ouest-africaine et de sa représentation dans les modèles est l'un des objectifs importants du programme AMMA. Le cycle de l'eau est le résultat d'interactions complexes entre l'atmosphère et le continent, dont les modalités sont très contrastées selon les échelles spatiales et temporelles, et les régions considérées. Les propriétés de la surface, du sol et du sous-sol conditionnent fortement la redistribution des précipitations sur le continent et la réalimentation de l'atmosphère, donnant lieu à des boucles de rétroaction encore mal comprises à ce stade. Les études de processus et les bilans d'eau établis à partir d'observations issues de la campagne AMMA, combinées à des produits de modélisation, nous permettent d'avancer sur la description et la compréhension de ces mécanismes. Il subsiste encore des incertitudes sur la quantification de certains processus notamment sur les liens entre l'évapotranspiration des surfaces, la végétation et les stocks d'eau souterrains. Au-delà de la compréhension du système de mousson, l'amélioration des modèles, de meilleures prévisions numériques en météorologie et le développement d'outils de quantification et de gestion des ressources en eau sont les principales applications attendues de ces travaux. e cycle de l'eau de la mousson d'Afrique de l'Ouest (MAO) résulte de l'interaction complexe entre des processus atmosphériques, océaniques et continentaux à diverses échelles spatio-temporelles. Parmi les nombreux objectifs du programme AMMA, l'amélioration de notre compréhension de ce cycle et notre capacité à pouvoir le simuler et le prédire sont indéniablement des objectifs majeurs autant d'un point de vue scientifique que sociétal.

L'étude de la composante atmosphérique du cycle de l'eau vise plus spécifiquement le transport de la vapeur d'eau et les interactions entre la circulation atmosphérique et les surfaces océaniques et continentales, et à décrire les mécanismes thermodynamiques et physiques conduisant à la formation des précipitations (Bock et al., 2011). La composante continentale s'intéresse au devenir de l'eau précipitée à travers les processus de surface (végétation, sols et sous-sols) qui sont conditionnés par les propriétés de la surface, le substrat géologique, le climat local. L'eau continentale est également une ressource vitale pour les écosystèmes et les besoins humains.

Selon les régions considérées, on rencontre une grande diversité dans le fonctionnement hydrologique aussi bien atmosphérique que continental, et selon les échelles spatiales et temporelles auxquelles on étudie le cycle de l'eau, les mécanismes dominants qui émergent peuvent en effet être différents. Une approche intégratrice pour analyser le fonctionnement de ce cycle et les couplages sous-sol/surface/ atmosphère consiste à évaluer des bilans d'eau dans chacun des compartiments et de les confronter à l'interface. L'analyse des bilans d'eau tente d'apporter, par une approche quantitative, un éclairage sur l'efficacité de processus (par exemple les quantités d'eau précipitée et évaporée) et les échanges nets entre compartiments (par exemple variation d'eau dans le sous-sol ou stockage/déstockage d'humidité dans l'atmosphère). Bien que l'exercice soit conceptuellement simple, les conclusions sont sensibles à la qualité des jeux de données utilisés. Le tableau 1 dresse un état des lieux des estimations des termes du bilan d'eau à la surface avant AMMA et des résultats de Meynadier et al. (2010a), utilisant les produits d'observation et de simulation de haute qualité issus d'AMMA sur la période 2002-2007. L'analyse révèle tout d'abord une forte variabilité, autant spatiale que temporelle, dans les quantités d'eau précipitée $\mathrm{P}$, évapotranspirée $\mathrm{E}$ et dans leur différence $\mathrm{E}$ - $\mathrm{P}$ qui représente le bilan à la surface. La moyenne et la variabilité des précipitations sont relativement cohérentes entre les études, ce qui n'est pas le cas pour l'évapotranspiration. Cette incohérence dans les estimations de E est apparue comme une source d'incertitude fondamentale dans l'interprétation du 


\section{Abstract}

The water cycle in the West African monsoon system

Improving our knowledge of the water cycle in the West African monsoon system and the way it is represented in numerical models is one of the major goals of the AMMA programme. The water cycle results from complex interactions between the atmosphere and the continent, with contrasted behaviour depending on the region and the space and time scales at which it is analysed. The properties of the surface, the soil and the sub-soil strongly drive water redistribution over the continent and towards the atmosphere, resulting in complex feed back loops that are still not fully understood. Process studies and water budgets computed from a mixture of observations and simulation products provided advances in the knowledge of these mechanisms. Some of the processes still remain uncertain, such as the links between evapotranspiration, vegetation and ground water storage. Beyond a better knowledge of the monsoon system, model improvement, better numerical weather predictions and the development of tools for water resources assessment and management are among the main applications of these studies. fonctionnement du cycle de l'eau à l'échelle régionale à partir des études passées. La mise en place d'un nouveau jeu de données utilisant des produits élaborés de meilleure qualité a permis de réduire de manière sensible l'incertitude sur les termes du bilan d'eau et d'en proposer une interprétation en accord avec le fonctionnement des processus atmosphériques et hydrologiques aux échelles inférieures.

Par les observations de terrain et les travaux de modélisation réalisés à l'échelle des bassins versants, le programme AMMA a permis des avancées significatives sur notre connaissance des mécanismes qui régissent le cycle de l'eau et sa sensibilité aux évolutions environnementales et aux fluctuations climatiques aux échelles pluriannuelles. On dispose maintenant des données nécessaires pour évaluer la représentation de ce cycle dans les modèles atmosphériques et hydrologiques et pour améliorer les simulations des transferts d'eau à la surface continentale et des échanges entre la surface et l'atmosphère notamment grâce au forçage et à l'assimilation de données. Le projet ALMIP (AMMA Land surface Model Intercomparison Project ; Boone et al., 2009), dédié à l'intercomparaison de modèles de surface sur la région ouest-africaine a joué un rôle central dans cette démarche. Cet article propose une synthèse des résultats obtenus, depuis l'analyse des bilans d'eau atmosphérique à l'échelle régionale jusqu'à l'étude des processus hydrologiques à l'échelle des bassins versants.

\section{Un fonctionnement hydrologique contrasté selon les régions et les échelles considérées}

\section{À l'échelle régionale}

Les précipitations dans le système de mousson sont caractérisées par un fort gradient méridien entre la région côtière au sud, où le cumul annuel dépasse les $1000 \mathrm{~mm}$, et le nord du Sahel où ce cumul atteint à peine $100 \mathrm{~mm}$ (figure 1). L'alternance des saisons est très marquée au Sahel où la saison des pluies est très courte (de juin à septembre). Plus près de la côte guinéenne, on distingue deux saisons de pluies, au printemps et à l'automne.

Tableau 1 - Bilans d'eau (en mm) établis par les études passées et périodes concernées, confrontés au jeu de données hybride développé par R. Meynadier (moyenne 20022007, domaines identiques aux autres études). Adapté de Meynadier (2010).

\begin{tabular}{|c|c|c|c|c|c|c|c|}
\hline \multirow[t]{2}{*}{ Référence } & \multirow[t]{2}{*}{ Domaine et période } & \multicolumn{3}{|c|}{ Résultats des études passées } & \multicolumn{3}{|c|}{ Résultats de Meynadier, 2010} \\
\hline & & $\mathbf{P}$ & $\mathbf{E}$ & E-P & $\mathbf{P}$ & $\mathbf{E}$ & $\mathbf{E}-\mathbf{P}$ \\
\hline $\begin{array}{l}\text { Cadet et Nnoli, } 1987 \\
\text { Août } 1979\end{array}$ & $\begin{array}{l}\text { Août } 1979 \\
10^{\circ} \mathrm{N}-12^{\circ} \mathrm{N} \text { x } 14^{\circ} \mathrm{W}-8^{\circ} \mathrm{E} \\
6^{\circ} \mathrm{N}-8^{\circ} \mathrm{N} \times 14^{\circ} \mathrm{W}-8^{\circ} \mathrm{E}\end{array}$ & $\begin{array}{l}210 \\
192\end{array}$ & $\begin{array}{l}300 \\
130\end{array}$ & $\begin{array}{r}90 \\
-62\end{array}$ & $\begin{array}{l}144 \\
243\end{array}$ & $\begin{array}{l}65 \\
87\end{array}$ & $\begin{array}{r}-79 \\
-156\end{array}$ \\
\hline $\begin{array}{l}\text { Brubaker et al., } 1993 \\
1963-1973\end{array}$ & $\begin{array}{l}7.5^{\circ} \mathrm{N}-15^{\circ} \mathrm{N} \times 10^{\circ} \mathrm{W}-15^{\circ} \mathrm{E} \\
\text { Moyenne annuelle } \\
\text { Moyenne août } \\
\text { Moyenne JAS }\end{array}$ & $\begin{array}{l}969 \\
234 \\
588\end{array}$ & $\begin{array}{r}1464 \\
232 \\
684\end{array}$ & $\begin{array}{r}495 \\
2 \\
96\end{array}$ & $\begin{array}{l}826 \\
205 \\
511\end{array}$ & $\begin{array}{r}642 \\
85 \\
260\end{array}$ & $\begin{array}{l}-184 \\
-121 \\
-251\end{array}$ \\
\hline $\begin{array}{l}\text { Gong et Eltahir, } 1996 \\
\text { 1992-1994 }\end{array}$ & $\begin{array}{l}5^{\circ} \mathrm{N}-15^{\circ} \mathrm{N} \times 10^{\circ} \mathrm{W}-15^{\circ} \mathrm{E} \\
\text { Moyenne août }\end{array}$ & 200 & 120 & -80 & 195 & 80 & -115 \\
\hline $\begin{array}{l}\text { Nicholson et al., } 1997 \\
\text { 1925-1985 }\end{array}$ & $\begin{array}{l}\text { Moyenne annuelle } \\
15^{\circ} \mathrm{N}-20^{\circ} \mathrm{N} \\
10^{\circ} \mathrm{N}-15^{\circ} \mathrm{N} \\
5^{\circ} \mathrm{N}-10^{\circ} \mathrm{N}\end{array}$ & $\begin{array}{r}244 \\
765 \\
1387\end{array}$ & $\begin{array}{r}244 \\
727 \\
1193\end{array}$ & $\begin{array}{r}0 \\
-38 \\
-194\end{array}$ & $\begin{array}{r}165 \\
638 \\
1312\end{array}$ & $\begin{array}{l}150 \\
514 \\
837\end{array}$ & $\begin{array}{r}-15 \\
-124 \\
-474\end{array}$ \\
\hline $\begin{array}{l}\text { Roads et al., } 2002 \\
\text { 1988-1999 }\end{array}$ & $\begin{array}{l}5^{\circ} \mathrm{N}-15^{\circ} \mathrm{N} \times 0-5^{\circ} \mathrm{E} \\
\text { Moyenne annuelle } \\
\text { Moyenne août }\end{array}$ & $\begin{array}{l}861 \\
195\end{array}$ & $\begin{array}{r}832 \\
90\end{array}$ & $\begin{array}{r}-29 \\
-105\end{array}$ & $\begin{array}{l}964 \\
166\end{array}$ & $\begin{array}{r}704 \\
82\end{array}$ & $\begin{array}{r}-260 \\
-84\end{array}$ \\
\hline $\begin{array}{l}\text { Fontaine et al., } 2003 \\
1968-2000\end{array}$ & $\begin{array}{l}\text { Moyenne août } \\
15^{\circ} \mathrm{N}-20^{\circ} \mathrm{N} \times 15^{\circ} \mathrm{W}-15^{\circ} \mathrm{E} \\
10^{\circ} \mathrm{N}-15^{\circ} \mathrm{N} \\
5^{\circ} \mathrm{N}-10^{\circ} \mathrm{N}\end{array}$ & $\begin{array}{r}7 \\
185 \\
240\end{array}$ & $\begin{array}{r}25 \\
120 \\
120\end{array}$ & $\begin{array}{r}18 \\
-65 \\
-120\end{array}$ & $\begin{array}{r}75 \\
202 \\
192\end{array}$ & $\begin{array}{l}47 \\
90 \\
71\end{array}$ & $\begin{array}{r}-28 \\
-112 \\
-121\end{array}$ \\
\hline Mme & Août : moyenne \pm écart-type & $183 \pm 74$ & $142 \pm 85$ & $-40 \pm 71$ & $178 \pm 51$ & $76 \pm 14$ & $-102 \pm 38$ \\
\hline moyeminte & Moyenne annuelle & 845 & 892 & 47 & 781 & 569 & -211 \\
\hline
\end{tabular}



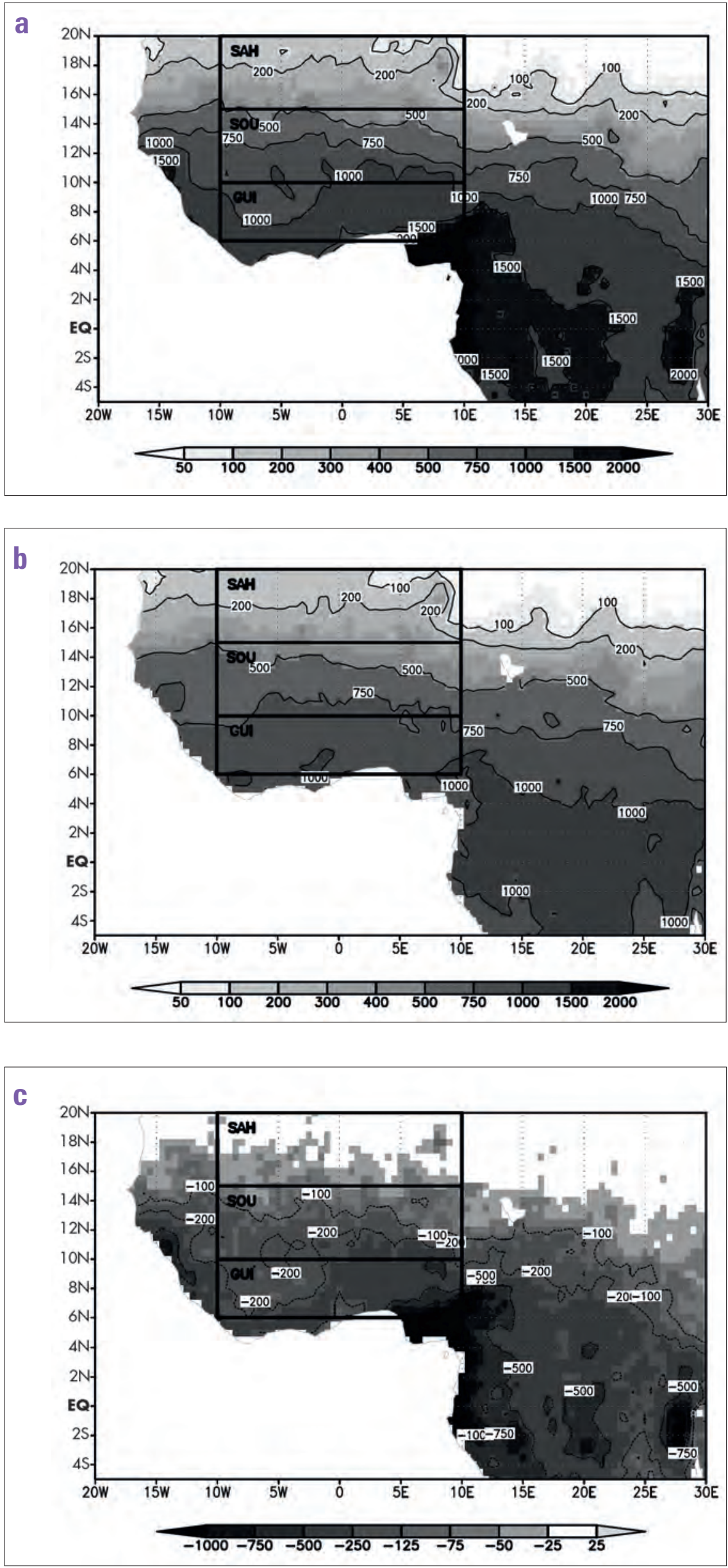

Figure 1 - Cumul annuel moyen sur la période 2002-2007 des précipitations TRMM (a), de l'évapotranspiration des modèles ALMIP (b) et de leur différence (c). Unités : mm. Extrait de Meynadier et al. (2010a).
Le gradient méridien de précipitation résulte d'interactions complexes entre la circulation atmosphérique sur la région (flux de mousson de sud-ouest) et la circulation océanique dans le golfe de Guinée (via des gradients de température de surface qui modulent fortement les flux verticaux d'humidité à l'échelle saisonnière). Les régions les plus arrosées sont marquées par un fort relief (monts de Guinée et du Cameroun). La réponse de la surface continentale d'un point de vue de l'évapotranspiration est fortement conditionnée par la distribution des précipitations. Le bilan E - P montre toutefois qu'il y a un net contraste entre les régions sahéliennes où il est presque nul (toute l'eau précipitée finit par retourner à l'atmosphère) et les zones plus humides (au sud de $15^{\circ} \mathrm{N}$ ) où un excès en eau est disponible à la surface. En moyenne annuelle, le stockage d'eau en surface et dans le sous-sol est pratiquement nul et l'excès d'eau est redistribué par les écoulements de surface.

La figure 2 apporte un éclairage sur le fonctionnement hydrologique à l'échelle régionale, le long du gradient méridien, grâce à l'analyse des relations entre les termes E, P, l'écoulement $\mathrm{R}=\mathrm{P}-\mathrm{E}$ et le rayonnement net à la surface $R_{\text {net }}$. Trois zones sont mises en relief. Dans la zone nord-sahélienne (15$\left.20^{\circ} \mathrm{N}\right)$, l'écoulement moyen est pratiquement nul et la relation entre $\mathrm{E}$ et $\mathrm{P}$ apparaît linéaire. Plus au sud, l'évapotranspiration atteint une limite maximale de l'ordre de $1000 \mathrm{~mm}$ qui est contrainte par la capacité évaporative des sols et de la végétation, et par l'énergie disponible pour transférer cette eau à l'atmosphère. Le rayonnement net est une mesure de cette énergie (Guichard et al., 2009), qui atteint son maximum dans la zone guinéenne $\left(120 \mathrm{Wm}^{-2}\right)$ où le surplus d'eau précipitée $(1000$ à $2000 \mathrm{~mm}$ ) alimente les écoulements.

\section{De la méso-échelle à l'échelle locale}

Les précipitations en Afrique de l'Ouest sont principalement engendrées par des systèmes convectifs de méso-échelle (voir dans ce numéro les articles de Guichard et al., p. 25-32 et Lafore et al., p. 11-16) qui traversent le souscontinent d'est en ouest. De ce fait, leur distribution spatiale à fine échelle est très hétérogène et intermittente (Lebel et Ali, 2009). Cette hétérogénéité subsiste à l'échelle annuelle, avec des différences de cumuls très importants sur de courtes distances (figure 3a). À méso-échelle, 


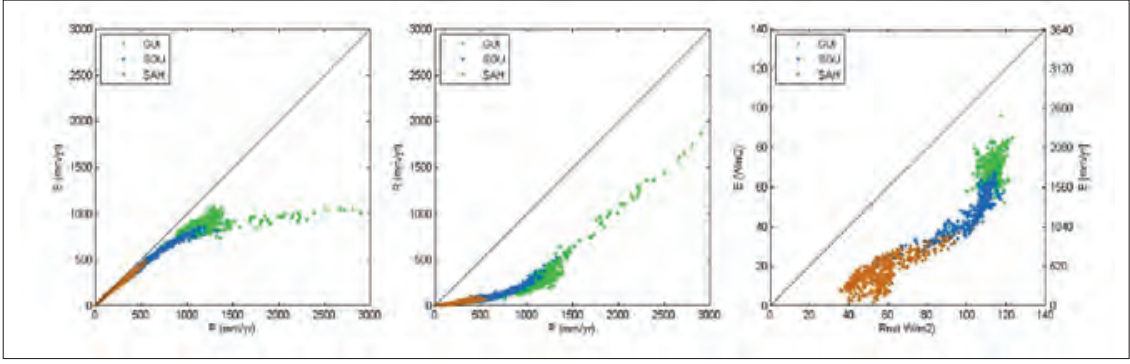

Figure 2 - Diagramme de dispersion spatiale des termes du bilan d'eau et du bilan d'énergie en moyenne annuelle dans les trois boîtes de la figure 1 (SAH, SOU, GUI) : chaque point représente un point de grille de la boîte correspondante. À gauche : E vs. P. Au milieu : R (écoulement) vs. P. À droite : $E$ vs. $R_{\text {net }}$ (rayonnement net à la surface). Adapté de Meynadier et al. (2010a).

le gradient méridien régional n'apparaît que lorsque l'on agrège les champs de précipitation sur de longues périodes (figure 3b). L'agriculture, très majoritairement pluviale dans la région, est sensible à ces inégalités spatiales des précipitations avec des conséquences sur la production agricole qui peuvent être dramatiques les années déficitaires.

La redistribution de l'eau à la surface continentale est très contrainte par la géomorphologie et les propriétés de la surface (perméabilité du sol et sous-sol, nature et densité de végétation, etc.), comme le montrent les exemples suivants pris sur les sites expérimentaux de 1'observatoire AMMA-CATCH (www.amma-catch.org ; Lebel et al., 2009). Au Mali, sur des sols superficiels rocheux, Timouk et al. (2009) montrent qu'à l'échelle de la saison, le ruissellement est la principale source de perte en eau et que l'évaporation reste faible ; le rayonnement solaire est principalement converti en chaleur sensible par échauffement de la surface et de l'air environnant. Au contraire, dans une forêt claire d'acacias saisonnièrement inondée, le rayonnement est totalement converti en chaleur latente. De plus, la quantité d'eau évaporée correspond à plus du double de la lame précipitée du fait des apports par ruissellement. Dans la région de Niamey, Ramier et al. (2009) mettent en évidence, au niveau local, le rôle du couvert sur le cycle de l'eau : infiltration plus profonde sur culture (mil) que sur jachère, en accord avec des travaux antérieurs (Peugeot et al., 1997) et évapotranspiration plus faible. L'eau stockée dans le sol est reprise par l'évapotranspiration en saison sèche. Comme dans toute la zone sahélienne, la propension des sols à l'encroûtement induit un ruissellement de surface important lors des averses. La fraction d'eau infiltrée est restituée à l'atmosphère par évapotranspiration en saison sèche, sauf dans les zones
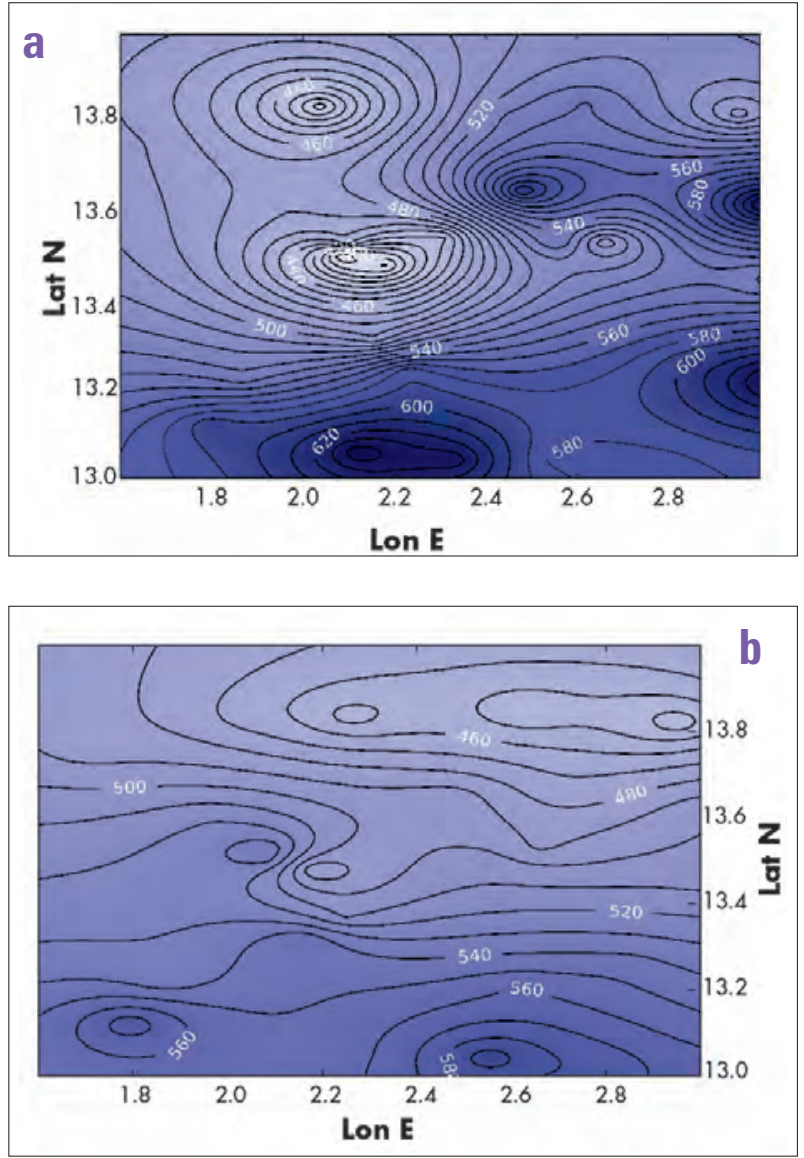

Figure 3 - Carte du cumul pluviométrique annuel en 2006 (a) et en moyenne sur 20 ans (1990-2009) sur la même zone au Niger (b).

d'accumulation (ravines, mares) où la percolation en profondeur permet la recharge de la nappe sous-jacente, située à une vingtaine de mètres de profondeur (Favreau et al., 2009). Cette nappe est très peu affectée par l'évaporation et subit une hausse continuelle depuis les années 1950.

Les conséquences à plus grande échelle de ces mécanismes locaux sont très contrastées selon les régions (Séguis et al., 2011a). Ainsi, dans la région de Niamey, en contexte géologique sédimentaire, le réseau hydrographique n'est plus fonctionnel actuellement, du fait de son entravement par des dépôts sableux liés à la forte aridité. La région se présente comme un chapelet de bassins versants indépendants de 1 à $10 \mathrm{~km}^{2}$ environ, parcourus par une série de ravines dont les exutoires sont des mares localisées aux points bas (Desconnets et al., 1997 ; Massuel et al., 2011). On parle de situation d'endoréisme ${ }^{(1)}$. On n'observe donc aucun écoulement aux échelles supérieures à celles de ces bassins versants, et, en particulier, aucun apport au fleuve Niger, et cela sur tout son parcours sahélien, en rive gauche. Dans la même région, mais en rive droite du fleuve Niger, en revanche, en contexte géologique de socle ancien, l'endoréisme est peu prononcé et le réseau hydrographique fonctionne. Pour des conditions climatiques équivalentes (BurkinaFaso et Niger, 400 à $600 \mathrm{~mm}$ ) mais un contexte géologique et géomorphologique différent, le comportement hydrologique est différentié. Descroix et al. (2009) ont proposé une cartographie des zones endoréiques et exoréiques à l'échelle de l'Afrique de l'Ouest.

Plus au sud, avec des précipitations plus abondantes (plus de $1000 \mathrm{~mm}$ ) et un couvert végétal plus dense, le contexte est clairement exoréique. Les transferts latéraux en sub-surface deviennent dominants par rapport aux transferts en surface dans l'alimentation des cours d'eau. Des nappes perchées saisonnières apparaissent et, contrairement au cas précédent, les nappes contenues dans la partie altérée et fracturée de la roche mère sont morcelées et montrent une dynamique de recharge-vidange saisonnière locale. Elles sont en général déconnectées des rivières et l'hypothèse privilégiée pour expliquer leur vidange saisonnière est la reprise profonde par les arbres (Séguis et al., 2011b; Descloitres et al., 2011 ; Guyot et al.,

(1) Du grec, littéralement " coule à l'intérieur 》, par opposition à exoréisme. 
2009, 2012). En effet, le système racinaire des ligneux peut descendre à plusieurs dizaines de mètres de profondeur. Sur un site de forêt au Bénin, des mesures de flux de sève (directement lié à la transpiration) montrent que les arbres transpirent à un taux relativement constant indépendamment du niveau des stocks souterrains, dès lors que leur feuillage est établi. Des expérimentations sont en cours pour estimer la profondeur à laquelle ces arbres extraient l'eau (Niger et Bénin). Par une approche statistique à l'échelle régionale sur 30 ans, Phillipon et Fontaine (2002) ont montré que les saisons des pluies excédentaires au Sahel sont associées à des stocks d'humidité continentale plus élevés en zone soudano-guinéenne à l'automne boréal précédent, qui perdurent en saison sèche. Les mesures piézométriques locales montrent que les niveaux minima et maxima des nappes réagissent aux excédents ou déficits de précipitation, avec un effet mémoire qui subsiste plusieurs mois (Séguis et al., 2011b). Les expériences décrites cidessus permettront de vérifier si l'on peut faire le lien entre la dynamique interannuelle des stocks souterrains, leur mobilisation par la végétation et les effets constatés à l'échelle régionale.

Les études à l'échelle régionale présentées sur la figure 1 mettent en évidence au premier ordre le contraste entre zones endoréiques au nord et exoréiques au sud. Mais les résultats aux échelles plus fines permettent de mieux appréhender les processus sous-jacents et la variété des situations. Le contraste entre les zones en rive droite (exoréisme) et gauche (endoréisme) du fleuve Niger à la latitude de Niamey est emblématique. De même, si le résidu $\mathrm{E}$ - $\mathrm{P}$ de la figure 2 correspond généralement à un écoulement, ce peut être également un terme de stockage souterrain (nappe de la région de Niamey).

\section{Sensibilité du cycle de l'eau aux fluctuations du climat et des conditions de surface}

L'Afrique de l'Ouest a connu une sécheresse sans précédent depuis les années 1970 avec des déficits de l'ordre de $20 \%$ en moyenne annuelle. Mais la réponse hydrologique à cette perturbation se manifeste de manière contrastée selon les régions considérées. Dans les domaines soudanien et guinéen (5 à $10^{\circ} \mathrm{N}$ ), on observe une diminution des écoulements des rivières de 40 à $60 \%$, avec une amplification du déficit pluviométrique, ainsi qu'une baisse du niveau des nappes (synthèse dans Descroix et al., 2009). Dans le domaine sahélien, en revanche, on observe une augmentation des écoulements (Mahé et Paturel, 2009), accompagné du développement du réseau de ravines (Leblanc et al., 2008). Dans les zones endoréiques, cela se traduit par des apports accrus aux mares et par l'apparition de nouvelles mares (Leblanc et al., 2008 ; Gardelle et al., 2010), ce qui explique la hausse du niveau de la nappe observée dans la région de Niamey (Favreau et al., 2009). Sur le fleuve Niger à Niamey, on observe ces dernières années un pic de crue en saison des pluies, inconnu auparavant (Descroix et al., 2009), correspondant aux apports significatifs des bassins en rive droite (au Niger et au BurkinaFaso). Les contributions de la rive gauche restent faibles (endoréisme). La cause principale de ce comportement apparemment contradictoire (appelé parfois le «paradoxe sahélien ») tient aux évolutions de la couverture végétale qui s'opèrent dans la même période. En effet, on observe un fort accroissement des surfaces cultivées dans toute la région (Leblanc et al., 2008). Le défrichement et la mise en culture de nouvelles terres favorisent l'érosion, l'encroûtement des sols et le ruissellement de surface (Séguis et al., 2004 ; Boulain et al., 2009). Cependant, la même tendance est observée dans la zone pastorale (nord du Sahel) où les surfaces cultivées sont pratiquement absentes et où la couverture végétale a fortement diminué pendant la période sèche en réponse au choc constitué par le déficit pluviométrique (Hiernaux et al., 2009a, 2009b). Qu'il soit d'origine anthropique ou climatique, le signal induit par l'évolution des surfaces (augmentation des écoulements des bassins versants) se substitue au signal pluviométrique (déficit). Les deux facteurs concourent à une réduction de l'apport en vapeur d'eau à l'atmosphère par évapotranspiration (Boulain et al., 2009). Cet exemple montre que les évolutions du cycle de l'eau à long terme ne peuvent s'étudier sans considérer à la fois les facteurs climatiques et environnementaux. Sur le domaine soudanien ( sud de $10^{\circ} \mathrm{N}$ ), le défrichement intense et la conversion des forêts et savanes en zones agricoles s'observent également sur la même période, mais les impacts respectifs de ces évolutions sur le cycle de l'eau et les écoulements n'a pas à ce jour été mis clairement en évidence.

\section{Bilans d'eau}

L'établissement de bilans d'eau permet de faire une synthèse de la connaissance de l'ensemble des compartiments du cycle de l'eau, en surface et dans l'atmosphère. On utilise pour cela une combinaison des meilleures données disponibles pour décrire chaque compartiment. À l'échelle régionale, seule l'imagerie satellitaire fournit des produits de précipitation comme, par exemple, $T R M M^{(1)}$ (figure 1). Cependant ces produits peuvent comporter des biais importants, notamment aux pas de temps journalier ou inférieur (Roca et al., 2010 ; Gosset et al., 2012). Ils sont néanmoins en constante amélioration (voir l'article d'Eymard et al., ce numéro, p. 80-89), et le lancement récent, en octobre 2011, du satellite franco-indien Megha-Tropiques est une nouvelle étape prometteuse. Les réseaux d'instruments à l'échelle régionale (GPS, radiosondages, par exemple) fournissent les données pour estimer les termes du bilan atmosphérique (voir équation 1 page suivante), notamment à l'aide de modèles numériques. Aux échelles plus fines, les réseaux de mesure au sol, tels que ceux déployés pendant AMMA (observatoire AMMACATCH et instruments associés), permettent de déterminer les termes du bilan de surface (écoulement, précipitations). Les observations d'évapotranspiration ne sont disponibles que très localement et les estimations spatialisées de E sont issues de simulations. Celles utilisées dans les études présentées ici proviennent du projet ALMIP, qui rassemble les simulations de 11 modèles de surface réalisées sur l'ensemble de l'Afrique de l'Ouest avec des forçages identiques. Ces simulations sont disponibles aux résolutions spatiales et temporelles de $0,5^{\circ}$ et 3 heures et sur la période 2002-2007 (Boone et al., 2009).

Les études réalisées à ce jour ne permettent pas encore de disposer de bilans intégrés surface-atmosphère aux mêmes échelles spatiales (Peugeot et al., 2011). Les approches par l'atmosphère concernent l'échelle régionale et celles menées à partir de la surface sont limitées à la méso-échelle, à l'exception des simulations ALMIP, mais elles ne sont pas validées régionalement pour

(1) TRMM : Tropical Rainfall Measuring Mission 
toutes les composantes du bilan de surface et souterrain. Deux exemples à ces deux échelles sont présentés ci-après.

\section{Approche par l'atmosphère}

La conservation de l'eau dans une colonne d'atmosphère s'étendant de la surface à son sommet $(\sim 15 \mathrm{~km})$ peut s'exprimer de manière schématique par l'équation suivante :

$\mathrm{E}-\mathrm{P}=\mathrm{MFD}+\mathrm{dPW}$

Cette équation fait intervenir deux termes à la surface ( $\mathrm{E}$ et $\mathrm{P}$ définis plus haut) et deux termes atmosphériques : la divergence des flux d'humidité (MFD), qui représente les exports nets d'humidité hors de la colonne par la circulation atmosphérique, et la tendance de l'eau précipitable (dPW), qui représente le taux de stockage de l'humidité dans la colonne. Ces deux quantités sont intégrées sur la colonne d'atmosphère. Le terme $\mathrm{dPW}$ est extrait de la réanalyse ERA-Interim avec une résolution journalière sur une grille de $0,75^{\circ}$ de côté. Le terme MFD est difficile à calculer explicitement et est de ce fait estimé par résidu de l'équation 1 . Sa précision dépend de la qualité des autres termes (E, P et dPW). Elle est d'environ 1 à $2 \mathrm{~mm} / \mathrm{j}$ (Meynadier et al., 2010a).

La figure 4 montre l'évolution saisonnière des termes de cette équation. Le terme $\mathrm{dPW}$, très faible, est nul en moyenne annuelle. Par conséquent, le terme MFD est très proche en moyenne de E - P. À l'échelle saisonnière, on observe ainsi que durant la saison des pluies, la quantité d'eau précipitée provient en parts pratiquement égales de l'évapotranspiration et de la convergence d'humidité $($ MFD $<0)$ dans l'atmosphère (dont une fraction non négligeable a pu être importée d'autres régions). À la fin de la saison des pluies, la surface continue à fournir de l'eau par évapotranspiration qui est exportée par la divergence d'humidité dans l'atmosphère (MFD > 0).

\section{Approche par la surface}

Comme précédemment, on définit pour un domaine tridimensionnel continental, par exemple le bassin versant d'une rivière incluant la surface et le sous-sol, l'équation de conservation de l'eau :

$\mathrm{P}-\mathrm{E}-\mathrm{R}=\mathrm{dS}$

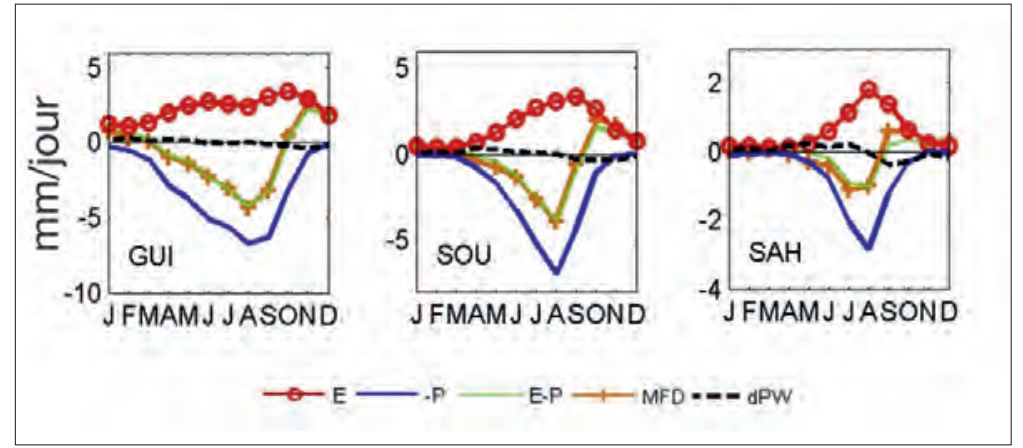

Figure 4 - Cycles saisonniers moyens des termes du bilan d'eau estimés sur la période 2002-2007 dans les trois boîtes (GUI, SOU et SAH) par l'analyse hybride. Adapté de Meynadier et al. (2010a).

où $\mathrm{P}$ et $\mathrm{E}$ ont le même sens que précédemment, $\mathrm{R}$ représente les exportations d'eau hors du système par les écoulements en rivière et $\mathrm{dS}$ représente la variation du stock total d'eau dans le bassin sur une période de temps donnée. Ce dernier terme correspond à la fois au stockage net dans la zone non saturée du sol et dans les nappes phréatiques. La figure 5 présente le bilan d'eau annuel (arrêté au $1^{\text {er }}$ mars) pour 2002-2007 sur le bassin versant de méso-échelle de l'Ouémé supérieur (10 $000 \mathrm{~km}^{2}$, au Bénin). Les termes P et $\mathrm{R}$ sont issus des observations de terrain (AMMA-CATCH) et E des simulations ALMIP. Le bilan est exprimé en fraction de la pluie.

L'évapotranspiration représente 65 à $85 \%$ des précipitations selon les années, et $75 \%$ en moyenne $(860 \mathrm{~mm})$, et les écoulements de surface $\mathrm{R}$ redistribuent 9 à $15 \%$ des pluies (en moyenne $11 \%$ soit $135 \mathrm{~mm}$ ). La pluie moyenne annuelle sur la période est de $1175 \mathrm{~mm}$. Le terme de stockage dS, calculé selon 1'équation 2 comme le résidu du bilan, représente en moyenne $15 \%$ de $\mathrm{P}(180 \mathrm{~mm})$, mais reste positif sur toute la période (figure 5). Cela suggère qu'il y aurait un stockage net d'eau dans le sous-sol chaque année, ce qui est contradictoire avec les observations disponibles sur la dynamique des nappes souterraines dans cette région. On peut observer une augmentation des stocks d'eau lors des années excédentaires en précipitations (2003 par exemple), mais qui est compensée par un déstockage lors des années déficitaires (2006), conduisant à une valeur de $\mathrm{dS}$ nulle en moyenne sur le long terme (Séguis et al., 2011). Deux hypothèses sont avancées pour expliquer ce défaut dans le bilan : la sous-estimation de l'évapotranspiration simulée par les modèles ALMIP ou la non prise en compte dans le bilan d'un terme de perte dû à un drainage à grande échelle par les aquifères.
Les résultats obtenus par Meynadier et al. (2010a) sur la même période aux points de grille contenus dans le bassin de l'Ouémé ( $\mathrm{E}-\mathrm{P}=-301 \mathrm{~mm}$, équation 1) sont cohérents avec les estimations issues de l'analyse à méso-échelle (E - $\mathrm{P}=-315 \mathrm{~mm})$. Les valeurs de $\mathrm{E}$ sont identiques (ALMIP), mais la différence s'explique par un biais (sec) sur les précipitations du produit TRMM par rapport aux mesures sol (-3\% en moyenne sur 2002-2007, mais qui peut fluctuer entre $-17 \%$ et $4 \%$ selon les années). Les simulations ALMIP ayant utilisé les pluies TRMM, les valeurs de E sont affectées par ce biais (pour les bilans de la figure 5 , les valeurs de $\mathrm{E}$ ont été corrigées de ce biais). La phase 2 du projet ALMIP permettra d'évaluer les termes calculés du bilan d'eau de manière plus fine que cela n'a été fait jusqu'à présent (Boone et al., 2009), en utilisant les mesures locales et de méso-échelle des termes des bilans d'eau et d'énergie, qui sont maintenant disponibles. L'existence d'un biais sur les valeurs calculées de E, les seules disponibles aux échelles moyenne à régionale, sera clarifiée.

Des progrès sont également attendus des nouvelles techniques de mesure, testées pendant la campagne AMMA, qui permettent de quantifier certaines propriétés du sous-sol et d'estimer les stocks d'eau. La gravimétrie mesure les variations de contenu en eau du sous-sol à partir des fluctuations qu'elles induisent sur le champ de pesanteur terrestre. Cette technique se déploie au sol à l'échelle locale (Hinderer et al., 2011), mais le satellite $G R A C E^{(1)}$ propose également des estimations à l'échelle globale (Tapley et al., 2004). Ces estimations ont été comparées sur l'Afrique de l'Ouest avec les simulations ALMIP (Grippa et al., 2011) et avec des mesures de déformation de surface par GPS (Nahmani et al., 2012). La résonance

(1) GRACE : Gravity Recovery And Climate Experiment. 


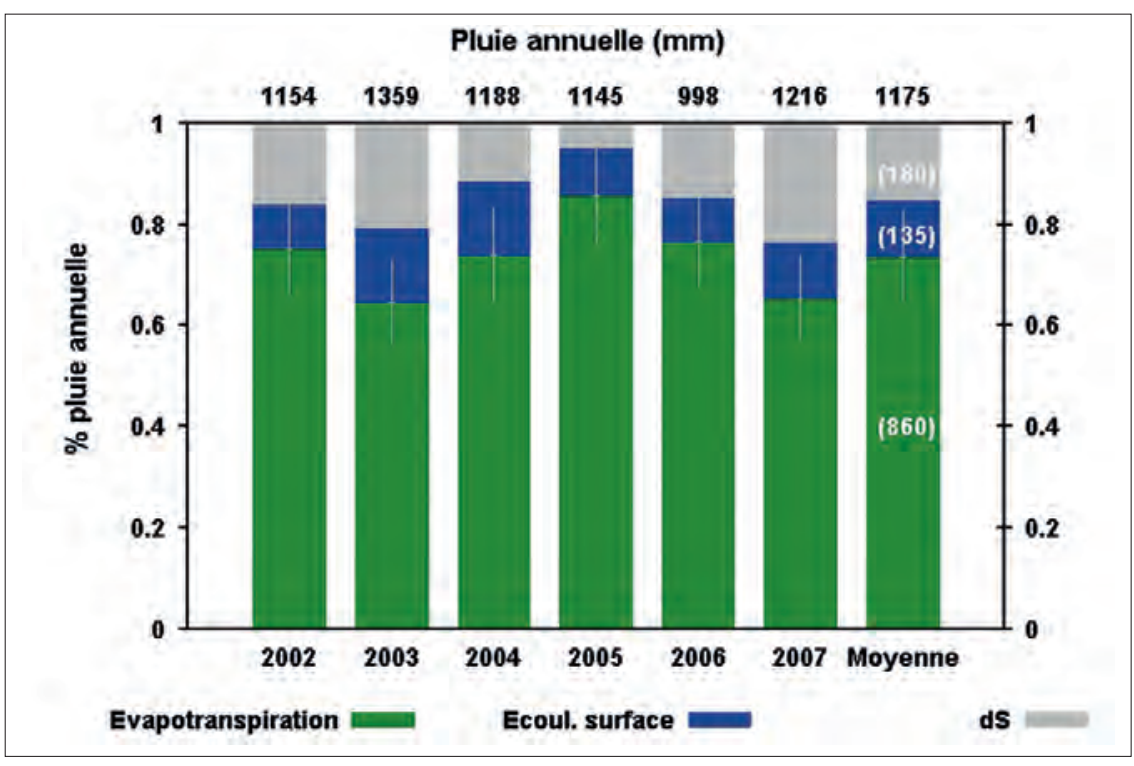

Figure 5 - Bassin de l'Ouémé supérieur (10 000 km², Bénin). Bilan annuel pour 6 années et bilan moyen sur la période exprimé en fraction de la précipitation. Cumul annuel de précipitations sur l'axe supérieur et valeurs en mm entre parenthèses.

magnéto-protonique (RMP) permet d'estimer quant à elle la teneur en eau et la porosité du milieu souterrain, et par conséquent de relier le volume stocké au niveau d'eau mesuré par les réseaux de piézomètres enregistrant le niveau des nappes. Par la combinaison de ces mesures, Boucher et al. (2009) et Pfeffer et al. (2011) montrent qu'il est possible de réduire la gamme d'incertitude sur l'estimation de la porosité. Au terme de ces études, l'on disposera de nouvelles données sur le cycle souterrain de l'eau qui nous permettront d'évaluer à quelle précision il est possible de boucler le bilan d'eau de surface.

Notre compréhension du cycle de l'eau n'est pas encore suffisante pour proposer des bilans cohérents à l'échelle régionale et à méso-échelle. Les résultats ci-dessus démontrent l'intérêt des approches à différentes échelles, du local au régional, et confirment la valeur essentielle des observations au sol. La confrontation de ces approches doit être poursuivie et approfondie pour progresser vers l'objectif d'une compréhension intégrée du cycle de l'eau dans le système de mousson.

\section{Un enjeu pour les applications}

\section{La prévision météorologique}

Les bilans d'eau établis à l'aide des simulations ALMIP forcées par les pluies TRMM (Huffman et al., 2007) sont probablement les plus fiables jamais établis à l'échelle régionale en Afrique de l'Ouest, même s'il reste difficile de valider ces produits à l'aide des seules observations AMMA disponibles (Boone et al., 2009). Pour la composante atmosphérique de ces bilans, les tendances d'eau précipitable ont été estimées à l'aide de la réanalyse ERAInterim (Simmons et al., 2006) et validées par les observations GPS (Bock et al., 2008 ; Meynadier et al., 2010a). L'estimation de la divergence des flux d'humidité, MFD, reste toutefois problématique à l'aide de modèles atmosphériques, qu'il s'agisse de modèles de prévision météorologique opérationnels ou de réanalyses (systèmes d'assimilation et de prévision utilisés pour simuler une période étendue dans une configuration fixe contrairement aux modèles opérationnels soumis à des améliorations régulières). $\mathrm{Ce}$ terme a, de ce fait, été estimé par résidu de l'équation 1 et a, par la suite, servi de référence pour évaluer la représentation du cycle de l'eau dans les modèles de prévision météorologique à l'échelle régionale (Agustí-Panareda et al., 2010b ; Meynadier et al., 2010b) et dans les modèles atmosphériques à méso-échelle (Guichard et al., 2010).

La figure 6 illustre la différence des termes du bilan d'eau moyen annuel simulés par trois réanalyses par rapport à la référence que constitue le jeu de données hybride (TRMM + ALMIP) sur la période 2002-2007. Il s'agit de la réanalyse ERA-Interim de 1'ECMWF ${ }^{(1)}$ (Simmons et al., 2006) et deux réanalyses du NCEP(2) : NCEP/DOE ${ }^{(3)}$ Reanalysis II (Kanamitsu et al., 2002) et
NCEP/NCAR ${ }^{(4)}$ Reanalysis I (Kalnay et al., 1996). On met en évidence un défaut commun à toutes en ce qui concerne les précipitations, avec une surestimation de celles-ci sur les massifs guinéens (au sudouest) et en Afrique Centrale, et une sous-estimation dans la région soudanosahélienne (au nord de $10^{\circ} \mathrm{N}$ ). Ce défaut peut être vu comme un positionnement trop au sud de la zone de précipitations sur le continent ouest-africain. Les travaux de Agustí-Panareda et al. (2010b) et Meynadier et al. (2010b) relient le blocage de la zone de pluies au sud à la présence d'une zone de divergence ou de convergence des flux d'humidité fortement réduite entre 10 et $15^{\circ} \mathrm{N}$. Les mécanismes conduisant à ce résultat sont complexes et font intervenir des couplages entre la circulation atmosphérique, le rayonnement et les paramétrisations dans les modèles atmosphériques. Deux défauts majeurs sont suspectés dans la représentation des processus au nord de cette région : 1) l'interaction des aérosols, présents en grande quantité, avec la dynamique via leur impact sur le bilan d'énergie et la circulation directe entre le flux de mousson au sud et la dépression thermique saharienne au nord, et 2) le lien trop faible entre la convection profonde et la convergence d'humidité. À cela s'ajoute un défaut dans la simulation des flux de chaleur latente (évapotranspiration) dont l'origine semble être connectée avec l'initialisation de l'humidité des sols dans cette région. Au final, le bilan $\mathrm{E}$ - $\mathrm{P}$ dans les réanalyses est en désaccord avec le jeu de données hybride, principalement au nord de $10^{\circ} \mathrm{N}$ où les réanalyses simulent un bilan positif (gain d'humidité pour l'atmosphère) alors que le bilan hybride indique un bilan nul, voire légèrement négatif (figure 1). Avec ces défauts mis en évidence, on explique en grande partie la forte disparité de résultats trouvés dans les études passées (tableau 1), principalement au Sahel. Il faut toutefois noter que si les dernières estimations de $\mathrm{E}, \mathrm{P}$ et $\mathrm{E}$ - P du tableau 1 sont proposées sur les mêmes zones géographiques que celles des travaux antérieurs, elles concernent des périodes différentes. Les effets de ces disparités d'échelles temporelles n'ont pas été étudiés, ce qui constitue une limite à l'exercice de comparaison.

(1) ECMWF : Centre européen pour les prévisions météorologiques à moyen terme (CEPMMT en français)

(2) NCEP : National Centers for Environmental Prediction.

(3) DOE : Department of Energy.

(4) NCAR : National Center for Atmospheric Research. 


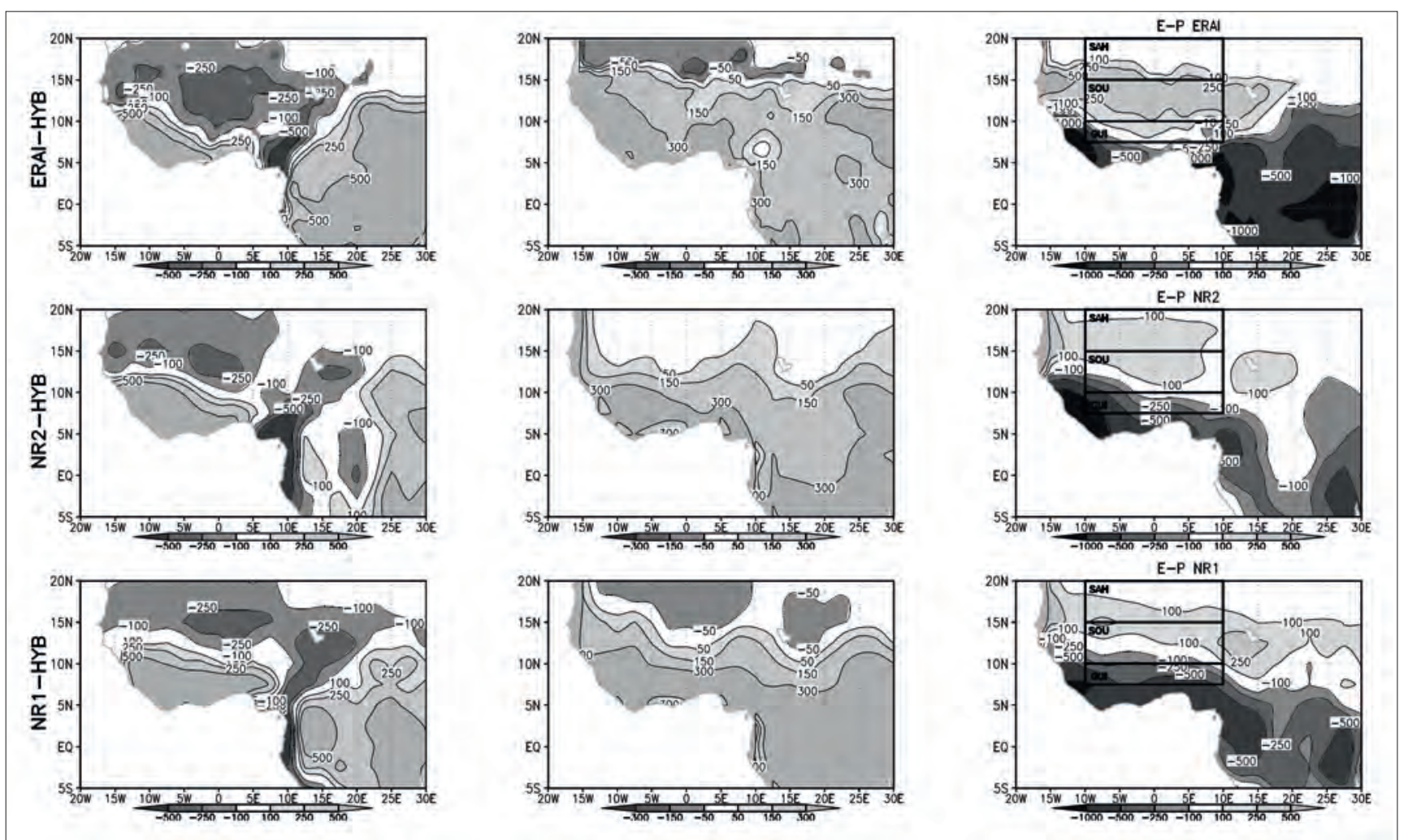

Figure 6 - Différence des moyennes annuelles des termes $P$ (à gauche) et $E$ (au milieu) extraits de trois réanalyses (ERA-Interim en haut, NCEP/NCAR réanalyse 2 au milieu et NCEP/NCAR réanalyse 1, en bas - voir texte) par rapport aux précipitations TRMM et aux évapotranspirations simulées par ALMIP. Moyenne annuelle de E - P (à droite) d'après les réanalyses. Extrait de Meynadier et al. (2010b).

L'étude de cas de Guichard et al. (2010), impliquant six modèles atmosphériques de méso-échelle, précise l'importance de la représentation des nuages dans ces modèles, via leur impact sur le rayonnement net à la surface et sur l'évapotranspiration. Elle confirme aussi le fort couplage existant entre l'eau précipitable et les précipitations. Ces travaux ouvrent de nombreuses perspectives d'amélioration des modèles atmosphériques.

\section{L'évaluation et la gestion des ressources en eau}

L'évaluation des ressources en eau exploitables et leur gestion durable constituent un enjeu important pour les politiques de développement des pays du Sud. La maîtrise de l'eau est considérée comme un levier pour le développement industriel et agricole ou pour la production d'énergie électrique. C'est également un enjeu pour l'approvisionnement en eau potable des populations. Les besoins en eau croissent avec le niveau de développement et la croissance démographique. Mais la menace du changement climatique et l'évolution de l'usage des terres avec la conversion des espaces naturels pour l'agriculture et l'urbanisation demandent de bien connaître les ressources exploitables et leur sensibilité aux changements globaux en cours.

L'étude du cycle de l'eau permet d'établir les connaissances scientifiques nécessaires à ces défis. L'eau utilisée pour les activités humaines est majoritairement d'origine souterraine, compartiment le moins bien connu et le plus difficile à étudier. Les propriétés des aquifères et leur mode de renouvellement ne sont pas toujours bien connus. Les techniques novatrices noninvasives (RMP, gravimétrie, voir cidessus) sont très intéressantes à ce titre, puisqu'elles permettent d'estimer les propriétés des aquifères à partir desquelles on peut déduire l'intensité des prélèvements qui garantit une exploitation durable. Associée aux observations, la modélisation du cycle de l'eau trouve des applications pour la gestion des ressources en permettant d'estimer, dans un cadre cohérent d'analyse, les termes du bilan qui ne peuvent être mesurés et leur dynamique sur une large gamme d'échelles de temps.

Dans le contexte des zones de socle où les aquifères sont peu capacitifs, face à la pression démographique, les ressources souterraines ne suffiront plus et il faudra recourir aux eaux de surface, dont la dynamique est plus facile à connaître que celle des eaux souterraines, puisque directement mesurable.

Cependant, les exemples décrits plus haut montrent que chaque compartiment du cycle de l'eau est sous la dépendance des autres, selon des modalités qui varient selon les contextes. Les conditions de surface et les facteurs d'origine climatique doivent être pris en compte conjointement, comme le montre l'exemple du paradoxe sahélien (moins de pluie, plus de ruissellement). Cela revêt une importance particulière pour les politiques de développement qui demandent des projections sur le long terme. La sensibilité des ressources en eau, sous toutes leurs formes, aux changements globaux est un enjeu essentiel, mais cela implique d'aborder de nombreuses questions de recherche et de lever des verrous scientifiques. Les tendances futures sur les précipitations sont moins claires dans les simulations climatiques sur l'Afrique de l'Ouest que celle concernant d'autres variables (températures de l'air par exemple). Par conséquent, l'impact hydrologique du changement climatique et les tendances sur les ressources sont difficiles à estimer. L'impact des conditions de surface sur le cycle de l'eau et les ressources doit être pris en compte. Les activités 
humaines constituent un forçage significatif sur la surface continentale. Pour estimer les ressources futures, on doit pouvoir construire des scénarios d'évolution du milieu à intégrer à la modélisation des impacts climatiques. Mais les processus à l'origine des interventions humaines sont liés aux relations complexes entre déterminants sociaux, micro et macro-économiques, culturels, climatiques qui pilotent les choix des individus et des sociétés. La constitution de scénarios socio-économiques est le pendant indispensable aux scénarios climatiques. La prise en compte des facteurs sociaux au même titre que les processus " géophysiques » dans des approches transdisciplinaires est un enjeu essentiel pour le futur.

\section{Conclusions et perspectives}

Les études menées au cours d'AMMA sur le cycle de l'eau de la mousson d'Afrique de l'Ouest ont permis de quantifier les termes du bilan d'eau atmosphérique et continental à différentes échelles spatio-temporelles, avec une précision accrue en comparaison aux études passées. Cette avancée a été rendue possible grâce à la collecte de nombreuses données d'observation et la réalisation de simulations avec de nombreux modèles dont la qualité ne cesse d'être améliorée. Néanmoins, les résultats présentés ici montrent que les estimations des bilans issus des analyses « par la surface » ou « par l'atmosphère » ne sont pas totalement cohérentes. Ces études ont précisé les incertitudes actuelles sur les estimations des quantités d'eau précipitée et évaporée par les sols et la végétation issues des systèmes d'observation et des modèles. Ces estimations doivent être mieux validées à partir des observations pour expliquer les termes résiduels qui subsistent dans les bilans. La représentation du cycle de l'eau souterraine, composante essentielle du bilan dans les modèles de surface doit être améliorée. Au-delà du système de mousson, cette évolution des connaissances constitue un enjeu pour l'estimation et la gestion des ressources en eau. Pour ce qui concerne les modèles atmosphériques, d'importants défauts ont été diagnostiqués dans la localisation et la quantité des précipitations et de l'évapotranspiration. Ils résultent d'interactions complexes entre la circulation atmosphérique et les processus radiatifs et thermodynamiques dans l'atmosphère et à la surface, et différentes hypothèses ont été explorées pour mieux en identifier les causes. Les études de processus et l'analyse fine des nombreuses observations disponibles après les campagnes de mesure d'AMMA permettront d'améliorer encore la qualité des modèles de prévision météorologique à moyen terme mais aussi les prévisions saisonnières et climatiques. En l'état actuel des connaissances, il faut donc être prudent en ce qui concerne l'interprétation et l'utilisation de modèles hydrologiques et atmosphériques couplés, même si les premiers résultats issus d'AMMA sont certainement plus fiables que ceux du passé. La base de données constituée pendant la campagne permettra de réaliser de nombreuses vérifications complémentaires, d'améliorer les modèles et de faire progresser les outils de simulations pour intégrer de mieux en mieux les composantes essentielles du cycle de l'eau.

\section{Remerciements}

Cet article a bénéficié des contributions de J. Demarty, S. Galle, M. Grippa, F. Guichard, L. Kergoat, E. Mougin, G. Quantin et A. Zannou. Que ces collègues en soient ici remerciés. 